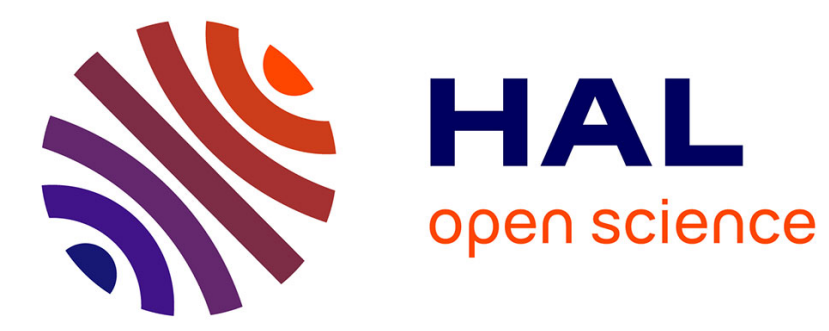

\title{
Review of solar irradiance forecasting methods and a proposition for small-scale insular grids
}

Hadja Maïmouna Diagne, Mathieu David, Philippe Lauret, John Boland, Nicolas Schmutz

\section{- To cite this version:}

Hadja Maïmouna Diagne, Mathieu David, Philippe Lauret, John Boland, Nicolas Schmutz. Review of solar irradiance forecasting methods and a proposition for small-scale insular grids. Renewable and Sustainable Energy Reviews, 2013, 27, pp.65 - 76. 10.1016/j.rser.2013.06.042 . hal-01090087

\section{HAL Id: hal-01090087 https://hal.science/hal-01090087}

Submitted on 3 Dec 2014

HAL is a multi-disciplinary open access archive for the deposit and dissemination of scientific research documents, whether they are published or not. The documents may come from teaching and research institutions in France or abroad, or from public or private research centers.
L'archive ouverte pluridisciplinaire HAL, est destinée au dépôt et à la diffusion de documents scientifiques de niveau recherche, publiés ou non, émanant des établissements d'enseignement et de recherche français ou étrangers, des laboratoires publics ou privés. 


\title{
Review of solar irradiance forecasting methods and a proposition for small-scale insular grids
}

\author{
Maïmouna Diagne ${ }^{1,2, *} \quad$ Mathieu David ${ }^{2} \quad$ Philippe Lauret $^{2}$ \\ John Boland ${ }^{3} \quad$ Nicolas Schmutz ${ }^{1}$ \\ ${ }^{1}$ Reuniwatt company \\ ${ }^{2}$ University of La Reunion \\ ${ }^{3}$ University of South Australia \\ *maimouna.diagne@reuniwatt.com
}

\begin{abstract}
Integration of solar energy into the electricity network is becoming essential because of its continually increasing growth in usage. An efficient use of the fluctuating energy output of photovoltaic (PV) systems requires reliable forecast information. In fact, this integration can offer a better quality of service if the solar irradiance variation can be predicted with great accuracy.

This paper presents an in-depth review of the current methods used to forecast solar irradiance in order to facilitate selection of the appropriate forecast method according to needs. The study starts with a presentation of statistical approaches and techniques based on cloud images. Next numerical weather prediction or NWP models are detailed before discussing hybrid models. Finally, we give indications for future solar irradiance forecasting approaches dedicated to the management of small-scale insular grids.
\end{abstract}

Keywords: Solar irradiance, forecast models, statistical models, NWP models, postprocessing methods.

\section{Introduction}

The contribution of photovoltaic systems (PV system) power production to the electric power supply is constantly increasing. Utility companies and transmission system operators have to deal with the fluctuating input from PV system energy sources. This is a new challenge compared with power production from conventional power plants that can be adjusted to the expected load profiles. An efficient use of the fluctuating energy output of PV systems requires reliable forecast information. Load patterns forecasted for the next 2 days provide the basis for scheduling of power plants and planning transactions in the electricity 
market in order to balance the supply and demand of energy and to assure reliable grid operation. These forecasts are used by utility companies, transmission system operators, energy service providers, energy traders, and independent power producers in their scheduling, dispatching and regulation of power.

In particular, insular territories experience an unstable electricity network and use expensive means in order to provide the power for the peak demand periods. Their grids are generally not interconnected with any continent and all the electricity must be produced inside the territory. The power of grid connected PV plants increases fast and can interfere with network stability. An efficient forecasting method will help the grid operators to better manage the electrical balance between demand and power generation. Kostylev and Pavlovski [1] identify three forecasting horizons (intra-hour, intra-day and day ahead) related to the grid operator activities (ramping events, variability related to operations, unit commitment, transmission scheduling, day ahead markets, hedging, planning and asset optimization).

Forecasting of global horizontal irradiance (GHI) is the first and most essential step in most PV power prediction systems. GHI forecasting approaches may be categorized according to the input data used which also determine the forecast horizon.

- Statistical models based on online irradiance measurements are applied for the very short term timescale from 5 minutes up to 6 hours (see Reikard, [2]). Examples of direct time series models are autoregressive (AR) and autoregressive moving average (ARMA) models. Furthermore, artificial neural networks (ANN) may be applied to derive irradiance forecasts.

- For short-term irradiance forecasting, information on the temporal development of clouds, which largely determine surface solar irradiance, may be used as a basis.

- Forecasts based on cloud motion vectors from satellite images (Lorenz and al, [3]) show good performance for the temporal range from 30 minutes up to 6 hours.

- For the subhour range, cloud information from ground-based sky images may be used to derive irradiance forecasts with much higher spatial and temporal resolution compared with the satellite-based forecasts.

- For longer forecast horizons, from about 4-6 hours onward, forecasts based on numerical weather prediction (NWP) models typically outperform the satellite-based forecasts (see Perez and al[4], Heinemann and al.[5]).

- There are also combined approaches that integrate different kinds of input data to derive an optimized forecast depending on the forecast horizon. 
Solar irradiance forecasts was assessed in terms of root mean square error (RMSE) and mean bias error (MBE or bias) which are defined as follows:

$$
\begin{aligned}
& R M S E=\sqrt{\frac{1}{n} \cdot \sum_{i=1}^{n}\left(x_{\text {pred }, i}-x_{o b s, i}\right)^{2}} \\
& M B E=\frac{1}{n} \cdot \sum_{i=1}^{n}\left(x_{\text {pred }, i}-x_{o b s, i}\right)
\end{aligned}
$$

where $x_{\text {pred,i } i}$ and $x_{o b s, i}$ represent the $i^{\text {th }}$ valid forecast and observation pair, respectively and $n$ is the number of evaluated data pairs. This metric are not formulate in the same way in all the papers we review. David and al [6] illustrated several formula wrongly called RMSE or MBE.

Many solar irradiance forecasting models have been developed. These models can be divided into two main groups: statistical models and NWP models. Statistical models are based upon the analysis of historical data. They include time series models, satellite data based models, sky images based models, ANN models, wavelet analysis based models, etc. NWP models are based on historical data and the reproduction of physical information.

The paper is organized as follow. In Section 2, statistical approaches are presented. In Section 3, cloud imagery and satellite based models proposed in the literature are reviewed. In Section 4, the NWP approaches presented in the literature are reviewed. In Section 5, hybrid models are evaluated. Finally Section 6 is dedicated to trends for future solar irradiance forecasting in an insular environment.

\section{Statistical models}

Forecasting methods based on historical data of solar irradiance are two categories: statistical and learning methods. Seasonality analysis, Box-Jenkins or Auto Regressive Integrated Moving Average (ARIMA), Multiple Regressions and Exponential Smoothing are examples of statistical methods, whilst AI paradigms include fuzzy inference systems, genetic algorithm, neural networks, machine learning etc.

\subsection{Linear models or time series models}

Statistical methods have been used successfully in time series forecasting for several decades. Using the statistical approach, relations between predictors, variables used as an input to the statistical model, and the variable to be predicted, are derived from statistical analysis. Several studies with respect to direct time series modeling have been performed. In Reikard [2], different time series models are compared. In Bacher and al.[7], the authors investigate the use of a simpler AR model to directly predict PV power in comparison with other models. 


\subsubsection{Persistence model}

It is useful to check whether the forecast model provides better results than any trivial reference model. It is worthwhile to implement and run a complex forecasting tool only if it is able to clearly outperform trivial models. Probably the most common reference model in the solar or wind forecasting community for short term forecasting is the persistence model. The persistence model supposes that global irradiance at time $t$ is best predicted by its value at time $t-1$ :

$$
\hat{X}_{t+1}=X_{t}
$$

The persistence model, also known as the naïve predictor, can be used to benchmark other methods. Persistence forecast accuracy decreases strongly with forecast duration as cloudiness changes from the current state. Generally, persistence is an inaccurate method for more than 1 hour ahead forecasting and should be used only as a baseline forecast for comparison to more advanced techniques.

In Perez and al.[4], the single site performance of the forecast models is evaluated by comparing it to persistence.

\subsubsection{Preprocessing of input data}

When using statistical time series analysis, any type of conditional forecast model is structured to deal with stationary series, at least weakly stationary. This means no trend nor seasonality, and the series is homoscedastic (constant variance). There are several ways to deal with non-stationary series to get them into an appropriate form.

2.1.2.1 Processes to obtain stationary solar irradiance time series The solar insolation is the actual amount of solar radiation incident upon a unit horizontal surface over a specified period of time for a given locality. It depends strongly on the solar zenith angle. For statistical models, it may be favorable to treat the influences of the deterministic solar geometry and the nondeterministic atmospheric extinction separately. For this purpose, two transmissivity measures have been introduced: clearness index $(\mathrm{k})$ and clear-sky index $\left(k^{*}\right)$.

2.1.2.1.1 Clearness index The clearness index $k$ is defined as the ratio of irradiance at ground level $I$ to extraterrestrial irradiance $I_{\text {ext }}$ on an horizontal plane:

$$
k=I / I_{\text {ext }}
$$

It describes the overall extinction by clouds and atmospheric constituents in relation to the extraterrestrial irradiance. This approach strongly reduces seasonal and daily patterns by considering the influence of the zenith angle, which is modeled by $I_{\text {ext }}$. The clearness index is widely applied to reduce the deterministic trend in irradiance time series. However, the clearness index accounts 
for only the trends caused by geometric effects on solar position. As atmospheric extinction depends on the length of the path of the radiation through the atmosphere, it is also governed by solar geometry.

2.1.2.1.2 Clear sky index The clear-sky index decreases with increasing of solar zenith angle. To account for this influence as well, the clear-sky index $k^{*}$ is introduced. $k^{*}$ is defined as the ratio irradiance at ground level $I$ to irradiance of a defined clear-sky model $I_{\text {clear }}$ :

$$
k^{*}=I / I_{\text {clear }}
$$

For the calculation of the clear-sky index, a clear-sky model and information on atmospheric input parameters are required. A clear sky model estimates the global irradiance, usually referred to as clear-sky irradiance $I_{\text {clear }}$, in clear sky conditions at any given time. An overview of different models is presented by Ineichen [8]. Clear-sky models range from empirical models to radiative transfer-based calculations. All these models need information on the state of the atmosphere as input.

The quantities introduced in this section are frequently used in solar modeling and forecasting. For example, some time series models explicitly require input parameters free of trend; hence, clearness index or clear-sky index can be an adequate choice. Also, satellite-based forecasts of irradiance are based on the concept of separately describing the influence of clouds and other atmospheric components by using the clear-sky index and a clear-sky model. Furthermore, most empirical models to derive the diffuse fraction of irradiance, necessary to calculate the irradiance on a tilted plane, are generally based on the clearness or clear-sky index.

Preprocessing of the input data can considerably contribute to improving the accuracy of forecasts, and different approaches are proposed. As mentioned earlier, stationary, trend-free time series are required for classical time series approaches, and might be beneficial also for ANN. Hence, the use of the clearsky or clearness index instead of irradiance data seems suitable. This approach is followed, for example, in Bacher and al.[7], and Kemmoku and al. [9]. On the other hand, Sfetsos and Coonick [10] argues that time series of the clearness or clear-sky index are mostly random, and hence do not provide a good basis for any learning algorithm. They recommend using irradiance values as input. Other examples of preprocessing of input data are the use of wavelets in Cao [11] and the use of the logarithm of irradiance values in Reikard [2].

2.1.2.2 Statistical tools for removing trend and seasonality Because of its unpredictable noise, it is not easy to find the trend in a day's series of solar irradiance. Several models exist to detrend the hourly solar irradiance (Baig and al.[12], Kaplanis [13]). Clear sky index, as described above, is one model to obtain the deterministic daily variation of irradiance. Fourier series is also a good predictor of the cyclical dependence of solar radiation, combining several significant frequencies in its depiction. Boland [14, 15] has shown that 
it captures yearly and intra-day cycles and can be used to effectively model the daily profile of solar irradiance time series, as well as capturing the variation over the year.

To judge the goodness of different detrending models, Ji and Chee [16] use the Augmented Dickey-Fuller (ADF) test to measure the stationarity of the detrended series. $\mathrm{ADF}$ is a test for unit root in a time series.

A time series possesses a unit root if it is a realization of the process

$$
X_{t}=\alpha X_{t-1}+Z_{t}
$$

where $\alpha=1$ and $Z_{t} \sim W N\left(0, \sigma_{Z}^{2}\right)$. WN indicates white noise, that is independent and identically distributed.

If there is a unit root in a time series, it is not stationary; otherwise, the time series may be considered stationary.

\subsubsection{ARMA model}

The Autoregressive Moving Average (ARMA) model is usually applied to auto correlated time series data. This model is a great tool for understanding and predicting the future value of a specified time series. ARMA is based on two parts: autoregressive (AR) part and moving average (MA) part. Also, this model is usually referred to as ARMA (p, q). In this p and q are the order of AR and MA respectively. The popularity of the ARMA model is its ability to extract useful statistical properties and the adoption of the well-known Box-Jenkins methodology $[17,15]$. ARMA models are very flexible since they can represent several different types of time series by using different order. It has been proved to be competent in prediction when there is an underlying linear correlation structure lying in the time series. One major requirement for ARMA model is that the time series must be stationary (see Hamilton [18]).

\subsubsection{ARIMA techniques}

The ARIMA (Auto-Regressive Integrated Moving Average) techniques (see Hamilton [18]) are reference estimators in the prediction of global irradiance field. It is a stochastic process coupling autoregressive component (AR) to a moving average component (MA), after differencing at appropriate time steps to remove any trends. It is in this way that ARIMA models allow treatment of non-stationary series.

Reikard [2] applies a regression in log to the inputs of the ARIMA models to predict the solar irradiance. He compares ARIMA models with other forecast methods such as ANN. At the 24-h horizon, he states that the ARIMA model captures the sharp transitions in irradiance associated with the diurnal cycle more accurately than other methods.

\subsubsection{CARDS model}

Jing et al [19] have developed a coupled autoregressive (AR) and dynamical system model to forecast solar radiation time series on hourly and intra-hourly 
time scales. The deseasoning was performed using Fourier series techniques as in Boland [14, 15]. The residual series formed by subtracting the Fourier series component from the original series cannot be modeled sufficiently using and autoregressive process alone. This is because the autoregressive process is too efficient at mean reversion to be able to reach the peaks in the series. The introduction of a resonating model introduced for the power market by Lucheroni [20] plus the judicious intermittent use of a proxy for curvature allows for a much superior fit to this residual series. The results found for the CARDS model compare very favourably with what Kostylev and Pavlovski [1] found from their survey of the literature. From that paper, the best performing model at the $1 \mathrm{~h}$ time step had a rRMSE of $17 \%$ for mostly clear days and $32 \%$ for mostly cloudy, whereas for the CARDS model it is $16.5 \%$ for all days.

\subsection{Non linear models}

For about one decade, there has been great interest in research on artificial intelligence (AI) techniques, not only for forecasting but also for a broad range of applications, including control, data compression, optimization, pattern recognition, and classification. An overview of the application of AI techniques for modeling and forecasting of the solar irradiance is presented in Mellit and Kalogirou,[21] where several approaches have been compared and analyzed.

\subsubsection{Artificial Neural Network (ANN)}

As an alternative to conventional approaches, ANNs have been successfully applied for solar irradiance estimation. ANNs recognize patterns in data and have been applied to solar forecasting. Using training data, ANNs reduce normalized root mean square error (rRMSE) of daily average GHI by as much as $15 \%$ when compared to 12-18 hour ahead NWP forecasts (see Guarnieri and al.[22]).

Heinemann and al. [5] use satellite images for horizons below 6 h; In Lorenz and al., [23] longer horizons of forecast produces by NWP models are used as input to an ANN to predict global irradiance.

Mellit and Pavan [24] developed a Multilayer Perceptron MLP-model to forecast the solar irradiance 24 hours ahead. The proposed model accepts as input parameters mean daily irradiance and mean daily air temperature; The output is solar irradiance data 24 hours ahead. Performance prediction of a grid-connected PV plant at Trieste, Italy, had a correlation coefficient of more than $98 \%$ for sunny days and slightly less than $95 \%$ for cloudy days.

Kemmoku and al, [9] used a multistage ANN to predict GHI of the next day. The input data to the network are the average atmospheric pressure, predicted by another ANN and various weather data of the previous day. Irradiance forecast by the multi-stage and the single-stage neural networks are compared with measured irradiance. The results show that the mean bias error (MBE) reduces from about 30\% (by the single-stage) to about 20\% (by the multi-stage).

Sfetsos and Coonick [10] use ANN to make one-step predictions of hourly values of global irradiance and to compare them with linear time series mod- 
els that work by predicting the clearness index. They introduced an approach for forecasting hourly solar irradiance using various artificial intelligence based techniques (ANN and ANFIS). They also investigated other meteorological variables such as temperature, wind speed, and pressure. A comparison between the various models in terms of prediction error and training time indicated that the network trained with the Levenberg-Marquardt algorithm (LM) network was as the optimum prediction model.

Mihalakakou and al., [25] developed a total solar irradiance time series simulation model based on ANN and applied it in Athens. The Neural Logic Network was identified as the model with the least error. It incorporates Logic Rules that produced an rRMSE of $4.9 \%$ lower than the persistence approach.

Fatih and al., [26] developed a time delay neural network (TDNN) model from general feed forward neural network to obtain the relationship between the input and output position in time series. Conventional ANN provides their response to the weighted sum of the current inputs. For TDNN, it extends the sum to a finite number of past inputs. In this way, the output provided by a given layer depends on the output of the previous layer's computed values based on the temporal domain of input values. Because of the very similar structure of the TDNN and the general MLP, back-propagation with some modifications can be applied to train the TDNN. The strength of this algorithm is its ability to model nonlinear series. With TDNN, there is no need to specify a particular model form, since the model is adaptively formed based on the features presented by the data. This data driven algorithm is suitable for many time series where no theoretical model is available.

\subsubsection{Wavelet Neural Network}

Mellit and al.[27] proposed an adaptive wavelet-network model for forecasting daily total solar irradiance. In this study, several structures have been investigated for resolving the missing data problem. In this particular estimation process, the model consists of an adaptive neural-network topology with the wavelet transformation embedded in the hidden units.

Cao and Lin, [28] proposed a new model for forecasting global solar irradiance based on diagonal recurrent wavelet neural network (DRWNN) and a special designed training algorithm. Simulation examples proved that the model is capable of mapping solar irradiance that is usually highly non-linear and time-changeable. This is because the DRWNN combines the advantages of both RNN (recurrent neural network) and wavelet neural network (WNN).

\subsubsection{ANN and classical time series models comparison}

A comparison of ANN and classical time series models has been carried out in Reikard [2] and in Sfetsos and Coonick [10]. Both studies find that the error of a simple regression model can be reduced considerably by a factor in the range of $0.6-0.8$ when using advanced models. Reikard [2] compares a regression model, the UCM Model, an ARIMA, a transfer function model, a neural network 
model and hybrid model. For this study, the author uses a logarithmic scaling of the input. Results show that for the resolutions of 60,30 and 15 minutes, the ARIMA model shows better results. In Sfetsos and Coonick [10], a feedforward ANN is identified as the most appropriate. The analysis in Reikard [2] for several stations with different climatic conditions also shows that there is a strong influence of the climatic conditions on both forecast accuracy and potential for improvement by the use of advanced models.

\section{Cloud imagery and satellite based models}

\subsection{Cloud imagery}

Besides the deterministic daily and annual patterns of irradiance, clouds cover as well as cloud optical depth have the strongest influence on solar irradiance at surface level. Clouds show a strong variability in time and space. Hence, determination of clouds at a designated time is an essential task in irradiance forecasting and modeling. For forecast horizons up to some hours, the temporal change of cloud structures is strongly influenced by cloud motion as a result of horizontal advection.

Satellites and ground-based sky images, have been used for the determination and forecasting of local solar irradiance conditions. The basis of this method relies upon the determination of the cloud structures during the previous recorded time steps. Extrapolation of their motion leads to a forecast of cloud positions and, as a consequence, to the local radiation situation. Satellites and ground-based sky images with their high temporal and spatial resolution offer the potential to derive the required information on cloud motion.

Through processing of satellite or ground images, clouds can be detected, characterized, and advected to predict GHI relatively accurately up to 6 hours in advance. The time series models based on satellite data and sky images detect the motion of cloud structures using motion vector fields (see Lorenz and al. $[3])$.

Irradiance for all sky conditions including cloudy skies may be derived using radiative transfer models (RTM) (see Heinemann and al. [5]) requiring input on the vertical structure of cloud physical parameters, for example, cloud and ice water content or droplet radius. Numerical weather prediction (NWP) models (see section 4) imply parameterizations of radiative transfer calculations.

The errors of satellite data and sky images based forecasts proposed in the literature increase drastically under low sun elevations, high spatial variabilities and low irradiance conditions. Hammer and al. [29] demonstrated achieved $17 \%$ rRMSE in satellite imagery for 30 minutes cloud index forecasts and $30 \%$ rRMSE at $2 \mathrm{~h}$ forecast horizons. For intra-day forecasts, a reduction in rRMSE by $7-10 \%$ compared to persistence forecasts was found.

Chow and al [30] presented a technique for intra-hour, sub-kilometer cloud shadow nowcasting and forecasting using a ground-based sky images for selected days at the UC San Diego. This technique allows one to obtain sky cover, cloud 
motion, cloud shadows, irradiance, and to forecast cloud locations.

\subsection{Satellite Images}

An approach to forecast solar irradiance based on Meteosat satellite images as a basis for PV power forecast was proposed in Lorenz and al. [31]. They investigated and compared various methods to derive motion vector fields from Meteosat data, and applied them to forecast solar irradiance up to some hours ahead. In Perez and al. [4], the results of irradiance forecasts based on the images of the Geostationary Operational Environmental Satellite (GOES) with a similar approach patterned after Lorenz and al, [3] are shown.

\subsection{Ground-based sky images}

To achieve high temporal and spatial resolution for intra-hour forecasts, NWP and satellite forecasts are currently inadequate. Ground observations using a total sky imager (TSI) present an opportunity to fill this forecasting gap and deliver a sub-kilometer view of cloud shadows over a large-scale PV power plant or an urban distribution feeder.

Compared with satellite data, ground-based sky images offer a much higher spatial and temporal resolution, including the possibility of capturing sudden changes in the irradiance, often referred to as ramps, on a temporal scale of less than 1 minute. The maximum possible forecast horizon strongly depends on the cloud speed and is limited by the time until the monitored cloud scene has passed the location or area of interest. This time is determined by the spatial extension of the monitored cloud scenes in combination with cloud velocities. In Chow and al [30], forecasts up to 5 minutes ahead were evaluated for 4 partly cloudy days. An estimation of a maximum possible extension of the forecast horizon in dependence on the cloud scene resulted in values ranging from 5 to 25 minutes.

Only short deterministic forecast horizons are feasible using a single TSI at a site due to low clouds and large clouds variabilities at the fine spatial scale studied. Capturing these features deterministically is nearly impossible with satellite or NWP approaches (Chow and al [30]).

\section{Numerical weather prediction models}

Numerical weather prediction (NWP) models are operationally used to forecast the state of the atmosphere up to 15 days ahead. The temporal development of the state of the atmosphere is modeled by the basic differential equations that describe the physical laws governing the weather.

Starting from initial conditions that are derived from worldwide observations, in a first step, the future state of the atmosphere is calculated with a global NWP model. Global NWP models are currently in operation at about 15 weather services. Examples are the Global Forecast System (GFS) run by the 
US National Oceanic and Atmospheric Administration (NOAA) and the European Centre for Medium-Range Weather Forecasts (ECMWF). Global models usually have a coarse resolution and do not allow for a detailed mapping of small-scale features, although resolution has increased rapidly during the last few years and nowadays, depending on the model, is in the range of $16-50 \mathrm{~km}$. In the next step, different concepts may be applied to account for local effects and to derive improved site-specific forecasts. One possibility is the downscaling by mesoscale models, which are also referred to as regional models. Mesoscale models cover only a part of the Earth but can be operated with a higher spatial resolution. They are routinely run by national weather services and private weather companies. Also, postprocessing methods, may be applied to model local effects. They allow the correction of systematic deviations in dependence on different meteorological parameters and for modeling of the irradiance if it is not

provided as output parameter of an NWP model. In the next few subsections we will review NWP configuration, input data feature, global model example, mesoscale model example, NWP accuracy, NWP limitation and postprocessing methods.

\subsection{NWP configuration}

Before running an NWP model, it should be configured. The principal variables to fix are the time step of internal calculation and the horizontal resolution of grid points.

\subsubsection{Temporal resolution}

The internal time step gives the period over which the change of the atmospheric variables is described by the dynamic equations. Temporal resolution of internal calculations in NWP models usually is considerably higher than that of the output variables. Output variables are delivered with a resolution of typically 1 hour for regional models and $3-6$ hours for global models. This internal time step may be down to 30 seconds for highly resolved calculations with mesoscale models and is about 10 minutes for global NWP models.

\subsubsection{Spatial resolution}

The horizontal resolution determines the spatial extent of weather phenomena that can be directly simulated. Grid points are usually distributed equally in the horizontal range. The resolution of global NWP models nowadays is in the range of $16-50 \mathrm{~km}$. In mesoscale models, the horizontal resolution may be down to $1 \mathrm{~km}$; weather services typically operate mesoscale models with a spatial resolution in the range of $5-20 \mathrm{~km}$. The resolution of vertical levels is generally adapted to the occurrence of physical processes that take place in certain regions of the atmosphere. 


\subsection{Input data features}

To start a forecast, information on the current state of the atmosphere is necessary. For global NWP models, this information is obtained from a worldwide network of meteorological observations and measurements. The key variables needed are the three-dimensional fields of wind, temperature, and humidity and the two-dimensional field of surface pressure. Boundary variables like snow cover or sea surface temperature are also of high importance. Regional models use initial conditions as well as lateral boundary conditions from global NWP model output, and also offer the possibility of integrating local measurements.

\subsection{Global model example: ECWMF}

ECMWF provides weather forecasts up to 15 days ahead, including solar surface irradiance and different cloud parameters as model output. ECMWF forecasts have shown their high quality as a basis for both wind and solar power forecasts. These forecasts are described here as an example of global NWP model forecasts.

The evaluations of ECMWF-based irradiance in Lorenz and al. [31], [32],[33]] are based on the T799 version with a spatial resolution of $25 \mathrm{~km} \mathrm{x} 25 \mathrm{~km}$. The current version T1279 was implemented in January 2010 and shows a horizontal resolution of $16 \mathrm{~km} \times 16 \mathrm{~km}$. Ninety-one hybrid vertical levels resolve the atmosphere up to $0.01 \mathrm{hPa}$ corresponding to approximately $80 \mathrm{~km}$. The temporal resolution of the forecasts is $3 \mathrm{~h}$ for the first 3 forecast days that are most relevant for PV power prediction.

\subsection{Mesoscale models example: MM5 and WRF}

The non-hydrostatic, fifth-generation mesoscale model MM5 has been developed at Pennsylvania State University and subsequently at the National Center for Atmospheric Research (NCAR). It uses a terrain-following coordinate, solves its finite-difference equations with a time-split scheme, and has multiple nesting capabilities (Grell and al. [34]. The WRF model is designed to be a flexible, state-of-the-art model and is developed as a collaborative effort of several institutes. WRF is supported as a community model with continuous development and integrates features of different mesoscale models, including also MM5 and the Eta model of the National Centers for Environmental Prediction (NCEP). In this sense, WRF can be seen as a follow-up model to MM5. The current WRF model, version 3, is described in Skamarock and al. [35]. Both WRF and MM5 offer a number of parameterizations for the different physical processes. This allows adapting the configuration of the model to the specific climatic conditions for an interested region. In addition, the capability of MM5 and WRF to integrate local measurements, for example, aerosols, may also contribute to improving forecast accuracy. The simulation of mesoscale and small-scale

phenomena, which is essential for calculations with high spatial resolution, is supported by the non-hydrostatic dynamics. With the possibility of high spatial resolution, the effects of topography may be considered in much more detail 
than for large-scale models.

\subsubsection{Input data}

Mesoscale models require input from global NWP models for initialization and boundary conditions. Frequently, GFS data of NOAA are used to initialize MM5 or WRF for operational applications, because, in contrast to ECMWF data, they are available for free. The input data used have a significant influence on the results, especially for cloudy conditions. This has been demonstrated in a case study reported in Heinemann and al. [5], where different NWP models (ECMWF and the global and local models of the German Weather Service (DWD)) were compared for initialization.

\subsubsection{Grid Nesting}

To achieve the intended high spatial resolution in a mesoscale model with reasonable computing time, the resolution of the driving global model is increased stepwise with internal nesting. For example, in the study reported in Heinemann and al. [5], the outer domain of MM5 covering large parts of Europe has a resolution of $27 \mathrm{~km} \times 27 \mathrm{~km}$, the next domain has a resolution of $9 \mathrm{~km} \times 9$ $\mathrm{km}$, and the final resolution of the innermost domain is $3 \mathrm{~km} \times 3 \mathrm{~km}$.

\subsubsection{MM5 and WRF configuration}

Recently, several research groups have investigated the potential of MM5 and WRF irradiance forecasts for solar energy applications. In a first step, an appropriate setup of MM5 or WRF has to be determined. Heinemann and al. [5] has addressed this task by comparing different configurations of the cumulus, moisture, and planetary boundary layer parameterizations with respect to irradiance calculations in a case study. To limit the computational effort when rerunning simulations for several configurations, a set of 6 test days was defined covering different cloud conditions: clear sky, broken clouds, and overcast.

Heinemann and al. [5], show an evaluation of MM5 forecasts for a 40day period in summer 2003 in Southern Germany in comparison with other forecasting approaches. Two studies comparing different methods to predict solar irradiance including WRF forecasts for different locations in the United States are reported in Remund and al. [36] and in Perez and al. [37]. In LaraFanego and al. [38], a detailed evaluation study of WRF irradiance forecasts in Andalusia (Southern Spain) is given with calculations of 1 month for each season.

\subsection{NWP model accuracy}

Forecasts beyond 6 hours, up to several days ahead, are generally most accurate if derived from NWP models. NWP models predict GHI using columnar (1D) radiative transfer models (RTM) (see Heinemann and al., [5]). Heinemann and al. [5] showed that the MM5 mesoscale model can predict GHI in clear skies 
without bias. However, the bias was highly dependent on cloud conditions and becomes strong in overcast conditions.

Perez and al. [39] examined the accuracy of the National Digital Forecast Database (NDFD), a derivative of the operational NWP models published by the NCEP. After a local correction function was applied, results show that for 8-26 h forecast horizons, the NDFD had an hourly-average GHI relative RMSE (rRMSE) of $38 \%$.

Remund and al. [36] evaluated different NWP-based GHI forecasts in USA, reporting rRMSE values ranging from $20 \%$ to $40 \%$ for a $24 \mathrm{~h}$ forecast horizon. Similar results were reported by Perez and al. [4], evaluating NWP-based irradiance forecasts in several places in the USA. Remund and al. [36] examined NWP biases compared to a single site and find that ECMWF and GFS next day GHI forecasts have a Mean Bias Error (MBE) of 19\%. This MBE was found to be approximately constant for intra-day (hour-ahead) to 3 days ahead forecast horizons.

Lorenz and al. [40] evaluated several NWP-based GHI forecasts in Europe. Overall, results showed rRMSE values of about $40 \%$ for Central Europe and about 30\% for Spain. Evaluating ECMWF accuracy in Germany, Lorenz and al. [31] showed that NWP MBE was largest for cloudy conditions with moderate clear sky index $\left(0.3<k t^{*}<0.6\right)$, while forecasted clear conditions were relatively unbiased. They reported rRMSE values of about $35 \%$ for single stations for a $24 \mathrm{~h}$ horizon forecasts.

\subsection{NWP Limitations}

A limitation of NWP forecasting is its coarse resolution. Even the $0.1^{\circ} \times 0.1^{\circ} \mathrm{NAM}$ spatial resolution is insufficient to resolve most clouds. Only an average cloud cover can be forecasted for a given point. For global models (GFS and ECMWF) the resolution is even coarser. However, even if the spatial resolution is finer, the temporal output intervals would not permit the assessment of time dependent cloud cover variability, important in predicting ramp rates and ranges of variability for solar power plants. Although NWP model time-steps are on the order of minutes, the RTM are run less frequently, and the output is only hourly (NAM) or every $3 \mathrm{~h}$ (GFS and ECMWF). Consequently, any patterns with characteristic time scales less than an hour are unresolved. Linking observed temporal variability in GHI to native NWP forecasts will require further research.

\subsection{Postprocessing methods}

Postprocessing methods are frequently applied to refine the output of NWP models. In particular, they may be utilized to:

- reduce systematic forecast errors (correction of systematic deviations);

- account for local effects (e.g., topography); 
- derive parameters that are not directly provided by the NWP models (e.g., solar surface irradiance is still not a standard output parameter);

- combine the output of different models in an optimum way.

Various approaches have been proposed to address these issues, some of which are presented in the following subsections.

\subsubsection{Model output statistics (MOS)}

MOS relates observed weather elements to appropriate variables (predictors) via a statistical approach. These predictors may be NWP model forecast, prior observations, or geoclimatic data.

A state-of-the-art MOS for solar irradiance predictions based on ECMWF forecasts has been introduced in Bofinger and Heilscher [41]. Multiple regression is applied to modify long-term monthly mean values of the forecasted data. Direct model output of ECMWF and statistically derived predictors are used to create daily solar electricity predictions accurate to $24.5 \%$ rRMSE for averaged daily forecasts. The MOS is operated on the basis of ground-measured irradiance values when available. For locations without irradiance measurements, irradiance derived from Meteosat data with the Heliosat method is used instead. A comparison of irradiance forecasts using this MOS scheme with WRF forecasts and other approaches is given in Heinemann and al. [5] and Lorenz and al. [31].

Lorenz and al. [31] related forecasted solar zenith angle (SZA) and clear sky index to ECMWF MBE for Germany, revealing a consistent over-prediction (up to $100 \mathrm{~W} . \mathrm{m} 2$ ) for moderately cloudy conditions. Using a MOS correction function eliminated bias and reduced RMSE of hourly forecasts by $5 \%$ for $24 \mathrm{~h}$ forecasts. A stepwise multivariate fourth-order regression was applied to derive the MOS correction function.

In Mathiesen and Kleissl [42] the analysis and MOS correction of GHI forecasts from NAM, GFS, and ECMWF models within the continental United States was presented. They indicated that MOS application to the NWP irradiance output was successful in minimizing bias and reducing RMSE, but did not provide information about bias source. MOS corrections in the measured clear sky regime did not reduce RMSE. This is because the MOS could not distinguish between RTM errors (over-prediction of GHI even for clear skies, especially for NAM) and cloud model errors (incorrect parameterization of RTM inputs). Consequently, many initially accurate forecasts were unnecessarily corrected. Differentiating between the sources of the error is important to selectively correct forecasts.

Although traditionally MOS schemes are mostly based on linear regression, any statistical approach relating observed variables to NWP output fits to the concept of MOS. In particular, ANN have also been used to improve NWP output with respect to irradiance prediction (Cao and Lin[43], Guarnieri and al.[22]). In Guarnieri and al.[22], an ANN is applied to irradiance forecasts of the NCEP Eta model run operationally at the Brazilian Center for Weather Forecasts and 
Climate Studies (CPTEC/INPE). An evaluation with measurements for two stations in the south of Brazil reveals a strong overestimation of the irradiance by the original forecasts, and a considerable improvement is achieved by the application of an ANN using different atmospheric forecast parameters of the Eta model as input.

\subsubsection{Kalman filter}

Systematic deviations of NWP output variables often depend on the meteorological situation. In Lorenz and al. [31], a bias correction in dependence on the predicted cloud situation for the application to ECMWF irradiance forecasts is introduced. A method for bias removal of irradiance forecasts using Kalman filtering is introduced and compared with the bias correction according to Lorenz and al. [31]. Kalman filters are designed to efficiently extract a signal from noisy data and are therefore expected to show a more robust performance if only limited training data are available, which is the case if the training is performed on the basis of individual stations.

Pelland et al. [44] found that the most suitable realization of their approach was a set of Kalman filter equations established separately for each forecast horizon and modeling the bias in dependence on the forecasted irradiance. The accuracy assessment was performed for single stations and for regional average values. At the level of individual stations, the bias removal based on Kalman filtering outperforms the other approach. However, the improvement compared with the original forecasts is small for single stations, while for regional averages both bias removal approaches significantly reduced the RMSE.

\subsubsection{Temporal interpolation}

Global model forecasts are provided with a temporal resolution of $3-6$ hours. The management of electricity grids, however, needs forecasts of the expected solar power input at least on an hourly basis. Different interpolation techniques may be applied in order to derive hourly forecasts from global NWP output. Lorenz and al. [31] propose an approach combining the forecast data with a clear-sky model to account for the typical diurnal course of irradiance.

\subsubsection{Spatial averaging}

Lorenz and al. [31] found that for ECMWF forecasts with an original spatial resolution of $25 \mathrm{~km} \times 25 \mathrm{~km}$ and temporal resolution of $3 \mathrm{~h}$, best results are achieved for average values of $4 \times 4$ grid points corresponding to a region of $100 \mathrm{~km} \times 100 \mathrm{~km}$ (Lorenz and al. [31]). However, the improvement compared with forecasts that evaluate only the next grid point is small due to the already coarse spatial and temporal resolution of the original forecasts.

Spatial averaging has a much stronger impact for mesoscale or multi-scale model output with hourly values and a finer grid resolution. An analysis of the high-resolution WRF forecasts presented in Heinemann and al. [5] showed that 
averaging irradiance predictions over an area of $180 \mathrm{~km} \mathrm{x} 180 \mathrm{~km}$ reduces the RMSE to approximately $85 \%$ of the RMSE when evaluating the nearest grid point only. Similar improvements are achieved for WRF forecasts provided by Meteotest that are delivered as average values of $10 \times 10$ model pixels, corresponding to an area of $50 \mathrm{~km} \times 50 \mathrm{~km}$ (Lorenz and al. [31]), and also for irradiance forecasts with the Canadian GEM model, where averaging areas in the range of $300 \mathrm{~km} \times 300 \mathrm{~km}$ to $600 \mathrm{~km} \times 600 \mathrm{~km}$ gave better results (Pelland et al. [44]). Mathiesen and Kleissl [42] report $100 \mathrm{~km} \times 100 \mathrm{~km}$ as a suitable averaging area for irradiance forecasts of GFS model and NAM model.

\subsubsection{Physical postprocessing approaches}

A few studies also investigate physical postprocessing procedures involving radiation transfer calculations. This allows for integrating additional parameters that are generally not modeled in detail with NWP models, for example, aerosols. A partly physical postprocessing procedure for topographic downscaling of solar irradiance forecasts in mountainous regions is proposed in LaraFanego and al. [38]. The disaggregation is carried out by accommodating the initial WRF irradiance estimates to the elevation of a target digital elevation model with a spatial resolution of $90 \mathrm{~m} \times 90 \mathrm{~m}$. The proposed method accounts for shading, sky-view reduction, reflected irradiance, and scaling to the inclined terrain surface.

\subsection{Human interpretation of NWP output}

Finally, a traditional method to obtain improved local forecasts from NWP model output is the participation of a human forecaster [16]. Meteorologists at weather services routinely analyze and compare the output of different global and local NWP models and meteorological measurements. In particular, they also use their expert knowledge to decide on the final forecast values, for example, of cloud cover. Solar irradiance forecasts may be derived by combining the cloud cover forecasts of meteorologists with a clear-sky model (see section 2.1.2.1.2). An advantage of this approach is that forecasts may be adjusted for local events or weather situations difficult to forecast with NWP models or statistical methods, like, fog.

\section{$5 \quad$ Hybrid models}

Hybrid models have been introduced to overcome the deficiency of using a individual model such as statistical methods (ARIMA, Multiple Regression and etc.) and AI methods. Hybrid models merge different methods to improve the prediction accuracy. Hybrid models can be also referred as combined models or ensemble models and often these terms are used synonymously. Hybrid methods can be implemented in three different ways; linear models, nonlinear models and both linear and nonlinear models. 
With the intention to improve the forecasting accuracy, the combination of forecasting approaches has been proposed by many researchers ([45], [11], [28], [16], [2]). From their studies, they indicate that the integrated forecasting techniques outperform the individual forecasts.

Artificial intelligence techniques, such as fuzzy logic and neural networks, have been used for estimating hourly global solar irradiance from satellite images. The results seem to point out that fuzzy logic and neural network models are better than regression models.

Cao and Cao in [45] and [11] developed a hybrid model for forecasting sequences of total daily solar irradiance, which combines ANN with wavelet analysis.

Cao and Lin[28] use an ANN (with a special designed training algorithm) combined with wavelets (based on diagonal recurrent wavelet neural network $($ DRWNN) ) to predict next day hourly values of global irradiance. Different types of meteorological observations are used as input to the models; among others the daily mean global irradiance and daily mean cloud cover of the day are forecasted.

Ji and Chee [16] use a hybrid model of ARMA and TDNN to improve the prediction accuracy. They suppose that the daily solar irradiance series is composed by linear and nonlinear components and use the ARMA model to fit the linear component and the TDNN model to find the nonlinear pattern lying in the residual. This hybrid model has the potential to harness the unique features and strengths of both models. It is more accurate than using the ARMA or TDNN models separately.

\section{$6 \quad$ Future solar irradiance forecasting approaches for small-scale insular grids}

A summary of the literature on solar irradiance forecasting models illustrated by Figure 1 and Figure 2 gives indications for future work. Figure 1 shows the classification of forecasting models based on spatial resolution of input data and temporal resolution of output or foreseen data. Figure 2 illustrates the relation between the forecasting horizons, the forecasting models and the related activities for grid operators.

However, a further consideration in choosing among forecasting models is efficiency. Reunion Island is a small territory with a high relief, a lot of microclimates and cloud formation processes. In this context, the selection of forecasting model is based on small horizontal and temporal resolution of forecasting model.

Based on these consideration, for day ahead and intra-days forecast horizon, we find that global models like ECMWF and GFS whom present generally reliable results are limited by their coarse resolution for Reunion Island. We suggest to use the mesoscale model WRF. It allows for small horizontal and temporal resolution. 


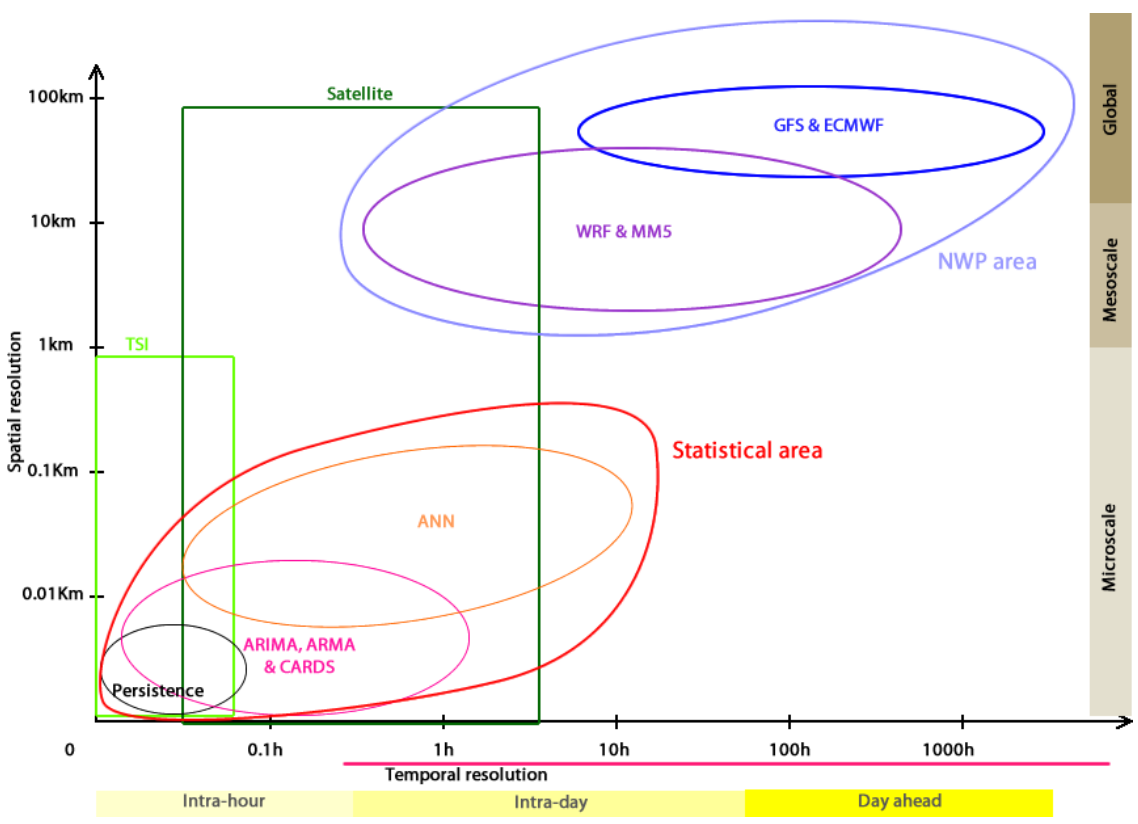

Figure 1: Classification of model based on spatial and temporal resolution

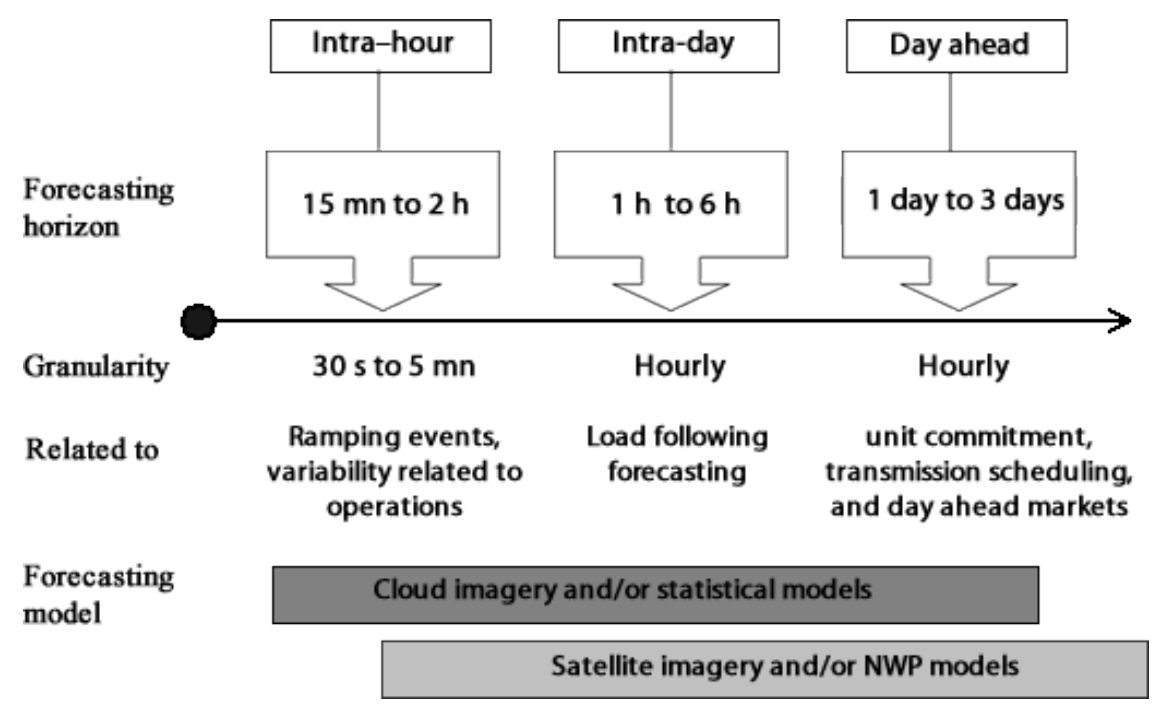

Figure 2: Relation between horizons, models and activities 
For intra-day and intra-hour forecast horizon time series models are used. The large number of data (GHI) measured on the ground offers a large set of temporal series of irradiance. This time series will permit building a statistical forecasting model. In this forecast horizon, the ARIMA models seem to be the most reliable model. They can provide a forecast in a fraction of a second on a personal computer. However, for an horizon of forecast of a few minutes, the persistence model achieves better accuracy than ARIMA models. In this sense, the choice of the model depends critically on the horizon of forecast. At longer horizons, the data are dominated by the diurnal cycle. In this case ARIMA models work better. At higher frequency, the data is more dominated by short-term patterns which can be picked up by persistence or ANN.

\section{Conclusions}

Solar irradiance forecasting is important for the integration of photovoltaic plants into an electrical grid. Proper solar irradiance forecasting helps the grid operators to optimize their electricity production and /or to reduce additional costs by preparing an appropriate strategy.

A number of time series models and numerical weather prediction (NWP) models have been reviewed in this paper. From the description of the various results of solar irradiance forecasting, we maintain that the choice of the appropriate forecasting models depends on forecast horizon and the available data. For forecast horizon from 6 hours up to 3 days ECMWF associated with a MOS post-process shows the most accurate results. However, in the case of Reunion Island, the WRF model seems to be more pertinent. For a smaller forecast horizons, from 5 minutes to 4 hours ARIMA seems to present the best accuracy. Cloud imagery and a hybrid model can improve the results of forecasting when solar irradiance presents a strong variability like in many of insular territories.

It is worth noting that Kostylev and Pavlovski [1] have done extensive analysis of the best performing models on differing time scales. As stated above, different models are best at different forecast horizons. They give the estimates of the best rRMSE values for both mostly cloudy times and mostly clear. The values range from approximately $17 \%$ at one hour, to $22 \%$ for three days for mostly clear. For mostly cloudy, they range from $33 \%$ to $44 \%$ over the forecast range of one to three days.

Future work will include several elements to improve forecast accuracy. Sky image techniques will be used to account for the process of cloud formation. The interesting methods identified here (WRF, ARIMA and AR) will be combined

to sky images to yield a comprehensive and more accurate forecast product with different horizons of forecast. The goal is to take care of the needs of grid operators. 


\section{Acknowledgements}

The authors would like to thank the Reuniwatt company for its support for the present work.

\section{References}

[1] A. Pavlovski V. Kostylev. Solar power forecasting performance towards industry standards. 1st International Workshop on the Integration of Solar Power into Power Systems Aarhus, 2011. Denmark, October.

[2] Gordon Reikard. Predicting solar radiation at high resolutions: A comparison of time series forecasts. Solar Energy, 83(3):342 - 349, 2009.

[3] E Lorenz, A Hammer, and D Heinemann. Short term forecasting of solar radiation based on satellite data. In EUROSUN2004 (ISES Europe Solar Congress), pages $841-848,2004$.

[4] Richard Perez, Sergey Kivalov, James Schlemmer, Karl Hemker Jr., David Renn, and Thomas E. Hoff. Validation of short and medium term operational solar radiation forecasts in the us. Solar Energy, 84(12):2161 - 2172, 2010 .

[5] D. Heinemann, E. Lorenz, and M. Girodo. Forecasting of solar radiation. In Solar Energy Resource Management for Electricity Generation from Local Level to Global Scale, pages 223-233. Nova Science Publishers, 2006.

[6] Mathieu David, Hadja-Mamouna Diagne, and Philippe Lauret. Outputs and error indicators for solar forecasting models. In Proceedings of the World Renewable Energy Forum 2012 (WREF 2012), Denver, USA, May 2012.

[7] Peder Bacher, Henrik Madsen, and Henrik Aalborg Nielsen. Online shortterm solar power forecasting. Solar Energy, 83(10):1772 - 1783, 2009.

[8] Pierre Ineichen. Comparison of eight clear sky broadband models against 16 independent data banks. Solar Energy, 80(4):468 - 478, 2006.

[9] Kemmoku Y, Orita S, Nakagawa S, and Sakakibara T. Daily insolation forecasting using a multi-stage neural network. Solar Energy, 66(3):193 199, 1999.

[10] A. Sfetsos and A.H. Coonick. Univariate and multivariate forecasting of hourly solar radiation with artificial intelligence techniques. Solar Energy, 68(2):169 - 178, 2000.

[11] J.C. Cao and S.H. Cao. Study of forecasting solar irradiance using neural networks with preprocessing sample data by wavelet analysis. Energy, 31(15):3435 - 3445, 2006. 
[12] A. Baig, P. Akhter, and A. Mufti. A novel approach to estimate the clear day global radiation. Renewable Energy, 1(1):119 - 123, 1991.

[13] S.N. Kaplanis. New methodologies to estimate the hourly global solar radiation; comparisons with existing models. Renewable Energy, 31(6):781 $-790,2006$.

[14] J. Boland. Time series analysis of climatic variables. Solar Energy, $55(5): 377-388,1995$.

[15] J. Boland. Time series and statistical modelling of solar radiation. In Recent Advances in Solar Radiation Modelling, pages 283-312. SpringerVerlag, 2008.

[16] Wu Ji and Keong Chan Chee. Prediction of hourly solar radiation using a novel hybrid model of arma and tdnn. Solar Energy, 85(5):808 - 817, 2011.

[17] George Box and Gwilym Jenkins. Time series analysis: Forecasting and control. Holden-Day, San Francisco, 1970.

[18] Bruce E. Hansen. Time series analysis james d. hamilton princeton university press, 1994. Econometric Theory, 11(03):625-630, 1995.

[19] Jing Huang, Malgorzata Korolkiewicz, Manju Agrawal, and John Bolandl. Forecasting solar radiation on an hourly time scale using a coupled autoregressive and dynamical system (cards) model. Solar Energy, (in press), 2013.

[20] C Lucheroni. A resonating model for the power market and its calibration. SSRN: http://ssrn.com/abstract=1850469, 2009.

[21] Adel Mellit and Soteris A. Kalogirou. Artificial intelligence techniques for photovoltaic applications: A review. Progress in Energy and Combustion Science, 34(5):574-632, 2008.

[22] RA Guarnieri, FR Martins, and EB Pereira. Solar radiation forecast using artificial neural networks. National Institute for Space Research, pages 134, 2008.

[23] E Lorenz, D Heinemann, and H Wickramarathne. Forecast of ensemble power production by grid-connected PV systems. Proc. 20th European PV, 2007.

[24] Adel Mellit and Alessandro Massi Pavan. A 24-h forecast of solar irradiance using artificial neural network: Application for performance prediction of a grid-connected pv plant at trieste, italy. Solar Energy, 84(5):807 - 821, 2010 .

[25] G. Mihalakakou, M. Santamouris, and D. N. Asimakopoulos. The total solar radiation time series simulation in Athens, using neural networks. Theoretical and Applied Climatology, 66(3-4):185-197, August 2000. 
[26] Fatih O. Hocaoglu, mer N. Gerek, and Mehmet Kurban. Hourly solar radiation forecasting using optimal coefficient 2-d linear filters and feedforward neural networks. Solar Energy, 82(8):714 - 726, 2008.

[27] A. Mellit, M. Benghanem, and S.A. Kalogirou. An adaptive waveletnetwork model for forecasting daily total solar-radiation. Applied Energy, 83(7):705-722, 2006.

[28] Jiacong Cao and Xingchun Lin. Application of the diagonal recurrent wavelet neural network to solar irradiation forecast assisted with fuzzy technique. Engineering Applications of Artificial Intelligence, 21(8):1255 - 1263, 2008.

[29] A. Hammer, D. Heinemann, E. Lorenz, and B. Lckehe. Short-term forecasting of solar radiation: a statistical approach using satellite data. Solar Energy, 67(1 - 3):139 - 150, 1999.

[30] Chi Wai Chow, Bryan Urquhart, Matthew Lave, Anthony Dominguez, Jan Kleissl, Janet Shields, and Byron Washom. Intra-hour forecasting with a total sky imager at the uc san diego solar energy testbed. Solar Energy, 85(11):2881 - 2893, 2011.

[31] Elke Lorenz, Johannes Hurka, Detlev Heinemann, and Hans Georg Beyer. Irradiance Forecasting for the Power Prediction of Grid-Connected Photovoltaic Systems. IEEE Journal of Selected Topics in Applied Earth Observations and Remote Sensing, 2(1):2-10, March 2009.

[32] Elke Lorenz, Thomas Scheidsteger, Johannes Hurka, Detlev Heinemann, and Christian Kurz. Regional pv power prediction for improved grid integration. Progress in Photovoltaics: Research and Applications, 19(7):757771, 2011.

[33] Elke Lorenz, Detlev Heinemann, and Christian Kurz. Local and regional photovoltaic power prediction for large scale grid integration: Assessment of a new algorithm for snow detection. Progress in Photovoltaics: Research and Applications, pages n/a-n/a, 2011.

[34] GA Grell, J Dudhia, and DR Stauffer. A description of the fifth-generation penn state/ncar mesoscale model (mm5). Technical Report 03, 1995.

[35] WC Skamarock, JB Klemp, J Dudhia, and al. A description of the advanced research wrf version 3. Technical Note NCAR/TN-475+STR. Boulder, CO: Mesoscale and Microscale Meteorology Division, National Center for Atmospheric Research, 2008.

[36] R. Perez J. Remund and E. Lorenz. Comparison of solar radiation forecasts for the usa. Proc.23rd European Photovoltaic Solar Energy Conference, 2:35,2008 . 
[37] Richard Perez, Mark Beauharnois, Elke Lorenz, Sophie Pelland, and Jim Schlemmer. Evaluation of numerical weather prediction solar irradiance forecasts in the us. ASES Annual Conference. Raleigh, NC, USA, 1721 May, 2011.

[38] V. Lara-Fanego, J.A. Ruiz-Arias, D. Pozo-Vzquez, F.J. Santos-Alamillos, and J. Tovar-Pescador. Evaluation of the wrf model solar irradiance forecasts in andalusia (southern spain). Solar Energy, 86(8):2200 - 2217, 2012. Progress in Solar Energy 3.

[39] Richard Perez, Kathleen Moore, Steve Wilcox, David Renn, and Antoine Zelenka. Forecasting solar radiation preliminary evaluation of an approach based upon the national forecast database. Solar Energy, 81(6):809 - 812, 2007.

[40] E. Lorenz, J. Remund, S.C. Mller, W. Traunmller, Steinmaurer, D. G., J.A. Ruiz-Arias, V.L. Fanego, L. Ramirez, M.G. Romeo, C. Kurz, L.M. Pomares, and C.G. Guerrero. Benchmarking of different approaches to forecast solar irradiance. In 24th European Photovoltaic Solar Energy Conference, 2009.

[41] Stefan Bofinger and Gerd Heilscher. "Solar electricity forecast approaches and first results". In 21st European Photovoltaic Solar Energy Conference, Dresden, number 0, pages 4-8, 2006.

[42] Patrick Mathiesen and Jan Kleissl. Evaluation of numerical weather prediction for intra-day solar forecasting in the continental united states. Solar Energy, 85(5):967 - 977, 2011.

[43] Jiacong Cao and Xingchun Lin. Study of hourly and daily solar irradiation forecast using diagonal recurrent wavelet neural networks. Energy Conversion and Management, 49(6):1396 - 1406, 2008.

[44] Sophie Pelland, George Galanis, and George Kallos. Solar and photovoltaic forecasting through post-processing of the global environmental multiscale numerical weather prediction model. Progress in Photovoltaics: Research and Applications, pages n/a-n/a, 2011.

[45] Shuanghua Cao and Jiacong Cao. Forecast of solar irradiance using recurrent neural networks combined with wavelet analysis. Applied Thermal Engineering, 25(23):161 - 172, 2005. 